\title{
Discontinuation of first-line bevacizumab in metastatic colorectal cancer: the BEAWARE Italian Observational Study
}

Tumori Journal

2019, Vol. 105(3) 243-252

(C) Fondazione IRCCS Istituto

Nazionale dei Tumori 2019

Article reuse guidelines:

sagepub.com/journals-permissions

DOI: $10.1177 / 0300891619834126$

journals.sagepub.com/home/tmj

@SAGE

\author{
Sara Lonardi', Guglielmo Nasti' , Daniele Fagnani \\ Donatello Gemma ${ }^{4}$, Libero Ciuffreda ${ }^{5}$, Cristina Granetto ${ }^{6}$, \\ Sara Lucchesi ${ }^{7}$, Alberto Ballestrero ${ }^{8}$, Maria Biglietto', \\ llaria Proserpio'0, Francesca Bergamo', Emanuela Proietti" ' \\ and Giuseppe Tonini ${ }^{12}$, on behalf of the BEAWARE study group
}

\begin{abstract}
Aims: BEAWARE investigated the pattern of first-line bevacizumab early interruption in the ltalian real-world setting of metastatic colorectal cancer.

Methods: A total of 386 patients were followed for 15 months after first-line chemotherapy + bevacizumab start. The rate of bevacizumab interruption for progression or adverse drug reactions (ADRs) constituted the primary endpoint.

Results: A total of $78.2 \%$ of patients interrupted bevacizumab: $56.6 \%$ for progression, $7.3 \%$ for ADRs, and $36.1 \%$ for other reasons. Median treatment duration was 6.7, 2.5, and 4.6 months, respectively. Median progression-free survival was 10.3 months; however, $35.8 \%$ of patients were not progressed and were thus censored at the data cutoff of 15 months, while $21.8 \%$ were still receiving bevacizumab. Patients discontinuing for progression/ADRs more frequently had metastases in $>$ I site $(p=.000 \mathrm{I})$, and a shorter median progression-free survival (6.9 vs I3.9 months, $p<.0001)$.

Conclusions: In Italy, first-line bevacizumab is interrupted mainly for progression, only $7.3 \%$ due to adverse events, and about one third of cases for other reasons. In clinical practice, the attitude to treat until progression as per guidelines might be implemented.

ClinicalTrials.gov Identifier: NCT0I609075
\end{abstract}

Keywords

Metastatic colorectal cancer, bevacizumab, therapy interruption, clinical practice, progression-free survival

Date received: I3 April 20I8; revised: 27 October 20I8; accepted: I8 December 2018

\footnotetext{
'UOC Oncologia Medica I, Istituto Oncologico Veneto-IRCCS, Padova, Italy

${ }^{2}$ Divisione di Oncologia Medica, Istituto Nazionale per lo Studio e la Cura dei Tumori "Fondazione Giovanni Pascale"-IRCCS, Naples, Italy ${ }^{3} \mathrm{UOC}$ Oncologia, ASST Vimercate, Italy ${ }^{4} \mathrm{UOC}$ Oncologia Medica, ASL Frosinone, Italy

5UOC Oncologia Medica I, AOU Città Della Salute e Della Scienza,

Torino, Italy

'UOC Oncologia Medica, AO Santa Croce e Carle, Cuneo, Italy

7 O Oncologia Medica, Ospedale Felice Lotti, Pontedera, Italy

${ }^{8}$ DIMI Università di Genova e Ospedale Policlinico San Martino, Genova, Italy
}

\footnotetext{
'UO Oncologia, A.O.R.N. 'A Cardarelli,' Napoli, Italy IOUO Oncologia Medica, ASST Sette Laghi, Varese, Italy

"Roche SpA Medical Affairs and CO, Monza, Italy

${ }^{12}$ OUC Oncologia, Policlinico Universitario Campus Bio-Medico, Rome, Italy

The complete list is provided in the Acknowledgment

Corresponding author:

Sara Lonardi, MD, SS Tumori Gastroenterici, UOC Oncologia Medica I, Istituto Oncologico Veneto-IRCCS, Via Gattamelata 64, 35I 37

Padova, Italy.

Email: sara.lonardi@iov.veneto.it
} 


\section{Introduction}

To date, colorectal cancer remains the second leading cause of cancer death in Europe. ${ }^{1}$ Approximately $20 \%-$ $25 \%$ of patients present metastases already at the time of diagnosis, and $50 \%-60 \%$ of the remainder will develop metastases later on. ${ }^{2}$ Although the introduction of new chemotherapy and biologic agents, used alone or in combination, has led to significant improvement in clinical outcomes, patient prognosis remains dismal and the 5-year survival rate is about $10 \%$.

Addition of bevacizumab has been shown to increase significantly the overall response rate $(44.8 \%$ vs $34.8 \%$, $p=.004$ ), overall survival (OS, 20.3 months vs 15.6 months, $p<.001$ ), and progression-free survival (PFS, 10.6 months vs 6.2 months, $p<.001$ ) in a randomized trial comparing the efficacy of 5-fluorouracil (5-FU)-based chemotherapy with or without monoclonal antibodies (mAbs). ${ }^{3}$ Further studies then confirmed the significant improvement of OS and PFS provided by bevacizumab in combination with oxaliplatin- and 5-FU-based chemotherapy in first-line regimens. ${ }^{4,5}$

Since its first approval by the Food and Drug Administration in 2004, new targeted agents have been developed for metastatic colorectal cancer (mCRC) (e.g. anti-epidermal growth factor receptor and other anti-vascular endothelial growth factor [VEGF] mAbs), but their use is either limited to subgroups of patients, such as those with a RAS wild-type (wt) tumor, ${ }^{6-8}$ or not recommended in first-line regimens. ${ }^{9}, 10$

As VEGF is physiologically produced, interruption of bevacizumab likely causes neoangiogenesis restoration, and this mechanism constitutes the rationale to continue its administration in maintenance therapy: indeed, patients with mCRC interrupting bevacizumab together with chemotherapy have been reported to have a significantly shorter PFS than those continuing therapy until progression of disease (PD) or toxicity (7.9 vs 10.4 months, $p<.0001){ }^{7}$ A phase III trial showed a significant improvement in PFS in patients treated with capecitabine and bevacizumab after induction with capecitabine plus oxaliplatin (XELOX) bevacizumab versus those randomized to observation (11.7 vs 8.5 months, $p<.0001$ ), suggesting the efficacy of the bevacizumab-based maintenance strategy. ${ }^{11}$ Yet the optimal maintenance regimen following induction therapy with chemotherapy plus bevacizumab remains controversial: recently, the AIO 0207 trial showed the noninferiority of bevacizumab alone compared to bevacizumab plus standard fluoropyrimidine in terms of time to failure of strategy (i.e. maintenance plus reinduction after first PD), ${ }^{12}$ whereas the SAKK trial failed to demonstrate the noninferiority of treatment holidays versus maintenance with bevacizumab alone. ${ }^{13}$ Analogously, 2 randomized phase III trials have recently shown that continuation or reintroduction of bevacizumab plus second-line chemotherapy beyond
PD leads to a significant extension in OS (of up to 1.8 months) compared to chemotherapy alone, ${ }^{14,15}$ supporting the idea of a benefit yielded by the prolongation of angiogenesis inhibition over time. However, despite this evidence and the fact that international guidelines by the National Comprehensive Cancer Network (NCCN) clearly suggest to continue bevacizumab until PD or unacceptable toxicity, data from clinical practice unveil a significant number of patients prematurely interrupting therapy for reasons other than PD. ${ }^{16,17}$ Besides progression, other causes include toxicity, chemotherapy holiday, achievement of maximum benefit, and physician decision. ${ }^{17}$ As a result, in US clinical practice, the median duration of first-line bevacizumab treatment was reported to be up to 5.4 months, although PD is expected $>10$ months after therapy initiation. ${ }^{16,17}$ In line with these results, data on file show that Italian physicians prematurely stop bevacizumab in daily practice, but this trend remains underinvestigated.

Here, we report the results of the BEAWARE observational study, which is the first Italian study specifically designed to assess, in the real-world setting, the proportion of patients with mCRC treated first-line with chemotherapy plus bevacizumab who discontinued therapy because of PD, adverse drug reactions (ADRs), or other reasons. Furthermore, BEAWARE provides a comprehensive evaluation of the causes for earlier interruption, together with the effects on PFS and the type of treatment adopted by patients who discontinued first-line therapy.

\section{Methods}

\section{Study design and patient population}

BEAWARE is an Italian multicenter, retrospective/prospective observational cohort study (ClinicalTrials.gov Identifier: NCT01609075) that collected data on patients with $\mathrm{mCRC}$ given first-line fluoropyrimidine chemotherapy plus bevacizumab and consecutively enrolled depending on their KRAS exon 2 mutational status. In order to obtain a representative sample of the target population, a consecutive enrollment considering KRAS exon 2 mutational status (according to a proportion wt:mut of 6:4) was suggested by study protocol based on published data. ${ }^{18}$ During the enrollment phase, this proportion was not satisfied in all centers, so the actual proportion was $49 \% \mathrm{wt}$ and $51 \%$ mut KRAS. Enrollment was performed by 74 centers specialized in the treatment of mCRC across Italy, between June 2012 and June 2013. Patients were eligible for this study if $\geqslant 18$ years of age, diagnosed with mCRC, and if they started a first-line fluoropyrimidine-based chemotherapy plus bevacizumab between September 1, 2011, and February 29, 2012. Other inclusion criteria were previous execution of KRAS genotyping test and informed consent and privacy forms given (authorization from the Privacy Guarantor was obtained for deceased or 
unreachable patients). Patients participating in clinical trials were excluded from the study. The total observational period was 15 months from the start of treatment with bevacizumab, thus the follow-up ended in June 2013. Patient inclusion was based on information present in medical records. Files from deceased patients meeting inclusion and exclusion criteria were allowed to participate in the study. For deceased subjects and for those starting bevacizumab greater than 15 months previously, all data were collected retrospectively. For alive patients starting bevacizumab less than 15 months previously, data were collected partly retrospectively, until the enrollment visit, and partly prospectively, until the 15 -month followup visit.

All patients provided informed consent prior to any study-specific procedures. Study approval was obtained first by the ethics committee of the coordinator center (Università Campus Bio-Medico, Rome) and then by the independent ethics committees at every institution (the full list is provided in the Acknowledgements). The study was conducted in accordance with the Declaration of Helsinki.

\section{Study endpoints}

The primary objective of this study was to investigate, in routine clinical practice, the proportion of patients who interrupted bevacizumab administration due to $\mathrm{PD}$ or to an $\mathrm{ADR}$ that requires discontinuation of the treatment as defined in the summary of product characteristics (SPC). Patients who died during treatment with bevacizumab contributed to this analysis and were considered as patients who interrupted bevacizumab for PD.

Secondary endpoints included evaluation of the proportion of patients who interrupted bevacizumab due to reasons other than PD or ADRs (not requiring discontinuation of treatment according to the SPC) and to describe those. The 2 groups of patients (who discontinued bevacizumab due to PD or ADRs related to the treatment and who discontinued for other reasons) were compared in terms of demographic and clinical characteristics, along with PFS. The proportion of patients undergoing second-line therapy, together with the regimens given, was also reported.

\section{KRAS genotyping}

Genomic DNA was extracted from tumor tissue blocks and screened for the presence of mutations in codons 12 and 13 of $K R A S$, according to each site's clinical practice at the time of observation.

\section{Data collection}

Data were collected with reference to information present in the medical records. All data were reported in a detailed structured case report form (CRF) in an electronic format (e-CRF). Each study investigator was responsible for collecting and reporting all sociodemographic, adverse reactions, and treatments data on the e-CRF. Collected data were verified during data entry by automatic controls (edit check). Any correction to original data entry was documented by means of an audit trail. Entered data were checked by data management staff, using automatic validation routines and database listings. Raised discrepancies were referred to the site's personnel for resolution. The database was locked on September 30, 2014.

All serious and nonserious adverse reactions related to bevacizumab, reported during the observation period, were included in the e-CRF.

\section{Clinical outcome assessment}

PFS was defined as the time elapsed from the start of bevacizumab treatment to the first PD or death. Patients who experienced PD (including death) were considered as failed observations, while other patients were considered as censored and were included in the analysis as well.

Time to ADR onset was calculated as the difference between the event occurrence date and the start date of bevacizumab treatment. In the case where a patient experienced more than one event, only the first ADR was considered. ADRs were coded according to the MedDRA Dictionary v. 16.1 .

\section{Statistical analysis}

Sample size was calculated on the basis of published data $^{4,5}$; thus, $33.3 \%$ of patients were expected to interrupt bevacizumab because of PD or an adverse reaction related to the drug. Sample size was calculated to have a relative error of the estimate equal to $15 \%$. A sample size of 420 enrolled subjects, corresponding to 336 evaluable patients assuming $20 \%$ as not evaluable, allowed to observe a $95 \%$ confidence interval (CI) of the expected proportion equal to $33.3 \pm 5.0 \%$. All patients evaluable for inclusion/exclusion criteria were considered for analysis.

\section{Results}

\section{Patients' characteristics}

Out of 437 patients with mCRC enrolled, 88.3\% (386/437) met inclusion criteria, while $11.7 \%$ (51/437) were excluded for protocol deviation or absence of privacy consent. At diagnosis, mean age was $63.6 \pm 10.8$ years. At the start of bevacizumab administration, $58.8 \%$ of all eligible patients were men and the mean age was $63.7 \pm 10.8$ years (Table 1 ). The Eastern Cooperative Oncology Group Performance Status (ECOG-PS) was $<2$ in $88.3 \%$ of patients. A total of $57 \%$ had metastases in one site $(56.8 \%$ with synchronous 
Table I. Patient characteristics at the time of bevacizumab start.

\begin{tabular}{|c|c|}
\hline Variable & $\begin{array}{l}\text { Eligible patients } \\
(n=386)\end{array}$ \\
\hline Age, y & $63.7 \pm 10.8$ \\
\hline Male & $227(58.8)$ \\
\hline \multicolumn{2}{|l|}{ Concomitant diseases } \\
\hline None & $159(4 \mid .2)$ \\
\hline Hypertension & $140(36.3)$ \\
\hline Diabetes & $34(8.8)$ \\
\hline Heart disease & $25(6.5)$ \\
\hline Dyslipidemia & $20(5.2)$ \\
\hline COPD & $13(3.4)$ \\
\hline Asthma & $4(1.0)$ \\
\hline Hepatitis C & $4(1.0)$ \\
\hline Hepatitis B & $3(0.8)$ \\
\hline Renal failure & $2(0.5)$ \\
\hline Cirrhosis of liver & I (0.3) \\
\hline Other & II4 (29.5) \\
\hline \multicolumn{2}{|l|}{ ECOG-PS } \\
\hline 0 & $254(65.8)$ \\
\hline 1 & $87(22.5)$ \\
\hline 2 & $8(2.1)$ \\
\hline 3 & I (0.3) \\
\hline NA & $36(9.3)$ \\
\hline \multicolumn{2}{|l|}{ Primary tumor site } \\
\hline Colon & $270(69.9)$ \\
\hline Rectum & $100(25.9)$ \\
\hline Colon-rectum & $14(3.6)$ \\
\hline Other ${ }^{\mathrm{a}}$ & I (0.3) \\
\hline NA & $\mathrm{I}(0.3)$ \\
\hline \multicolumn{2}{|l|}{ Organs with metastases } \\
\hline 1 & $220(57.0)$ \\
\hline$>1$ & $166(43.0)$ \\
\hline KRAS mut & $196(50.8)$ \\
\hline \multicolumn{2}{|l|}{$\begin{array}{l}\text { Concomitant chemotherapy } \\
\text { regimen }\end{array}$} \\
\hline FOLFIRI & $159(4 \mid .2)$ \\
\hline FOLFOX $(4,6,7)$ & $116(30.1)$ \\
\hline XELOX/CAPOX & $63(16.3)$ \\
\hline XELIRI/CAPIRI & $18(4.7)$ \\
\hline Capecitabine (monotherapy) & $12(3.1)$ \\
\hline FOLFOXIRI/FIr-B/FOx & $8(2.1)$ \\
\hline DeGramont & $6(1.6)$ \\
\hline 5-FU/FA (LV) bolus & $4(1.0)$ \\
\hline
\end{tabular}

Data are reported as mean \pm standard deviation or $n(\%)$.

aPrimary tumor localized in the intestine.

SD: standard deviation; COPD: chronic obstructive pulmonary disease; ECOG-PS: Eastern Cooperative Oncology Group Performance Status; NA: not available; mut: mutated; FOLFIRI: folinic acid, 5-fluorouracil (5-FU), irinotecan; FOLFOX: folinic acid, 5-FU/oxaliplatin; XELOX/ CAPOX: capecitabine, oxaliplatin; XELIRI/CAPIRI: capecitabine, irinotecan; FA: folinic acid; LV: leucoverin; FOLFOXIRI: folinic acid, 5-FU: oxaliplatin, irinotecan; FIr-B/FOx: triplet chemotherapy plus bevacizumab.

metastases, 36.4\% with metachronous metastases, and $6.8 \%$ with type not available, data not shown), whereas the remaining $43 \%$ had more than one organ involved $(56.6 \%$ with synchronous metastases, $38.6 \%$ with metachronous metastases, and $4.8 \%$ with type not available, data not shown). Metastases were most frequently found in liver (67.1\%), lung (30.8\%), abdominal lymph nodes (19.9\%), and peritoneum (15.5\%). KRAS exon 2 was mutated in 196 out of 386 patients $(50.8 \%)$.

\section{First-line treatment}

Regimen characteristics. The median time from mCRC diagnosis to the beginning of bevacizumab was 1.5 months (range 0-21.6). A similar percentage of patients received the combination of oxaliplatin-based and irinotecan-based doublets $(46.4 \%$ and $45.9 \%$, respectively), but with different proportions of intravenous and oral fluoropyrimidine (FP): FOLFOX/XELOX 30.1\% (116/386) and 16.3\% (63/386), respectively, and FOLFIRI/XELIRI $41.2 \% \quad(159 / 386)$ and $4.7 \%$ (18/386), respectively (Table 1). Only $5.7 \%$ of patients $(22 / 386)$ received FP monotherapy in association with bevacizumab, and $2 \%(8 / 386)$ were treated with a triplet plus bevacizumab. The median number of cycles administered was 11 (range 1-37) for chemotherapy (independently of bevacizumab) and 12 (range 1-35) for bevacizumab; the median duration of $\mathrm{mAb}$ administration was 7.2 months (range 0-16.5).

Treatment interruption and response. At the end of the observational period, only 31 patients $(8 \%)$ were still under treatment, while the majority of evaluable patients had interrupted chemotherapy $(92 \% ; 355 / 386)$. Bevacizumab was interrupted in $78.2 \%(302 / 386)$ of all cases (Table 2): due to PD occurrence in $44.3 \%$ (171/386) (including 74 deceased patients), to ADRs related to bevacizumab (requiring discontinuation of treatment according to the SPC) in $5.7 \%(22 / 386)$, and to other motivation in $28.2 \%$ (109/386). Considering only the subgroup of patients who discontinued $(n=302)$, the percentages were 56.6, 7.3, and 36.1, respectively. A detailed list of ADRs is provided in Table 3. Reasons other than PD or ADRs are heterogeneous (Table 4) and include toxicities related to bevacizumab that did not require interruption according to the SPC (grade 2 headache and grade 2 hemorrhage in 1 case each) and to chemotherapy (3 cases).

Overall, upon first-line therapy, PD occurred in $64.2 \%$ (248/386) of cases, with a median PFS of 10.3 months (95\% CI 9.2-11.2 months); the best response was CR in $8.3 \%(32 / 186)$ of subjects, partial response in $32.4 \%$ $(125 / 386)$, stable disease in $33.2 \%(128 / 386)$, and PD in $18.4 \%(71 / 386)$.

Comparisons between the subgroups of patients who interrupted bevacizumab. We next compared the main characteristics of patients subgrouped based on reasons for 
Table 2. Proportion of patients with metastatic colorectal cancer who discontinued first-line bevacizumab and reasons for interruption.

\begin{tabular}{llc}
\hline Reason for bevacizumab interruption & $\begin{array}{l}\text { Eligible patients } \\
(n=386), n(\%)\end{array}$ & $\begin{array}{l}\text { Patients interrupting } \\
\text { therapy }(n=302), n(\%)\end{array}$ \\
\hline Overall interruptions & $302(78.2)$ & $302(100.0)$ \\
PD or ADRs ${ }^{\text {b }}$ (primary endpoint) & $193(50.0)$ & $193(63.9)$ \\
PD & $171(44.3)$ & $171(56.6)$ \\
ADRs & $22(5.7)$ & $22(7.3)$ \\
Other reasons (secondary endpoint) & $109(28.2)$ & $109(36.1)$ \\
\hline
\end{tabular}

aPD as a motivation includes also deceased patients $(n=74)$.

bThe primary endpoint of the study considers only the ADRs requiring discontinuation of treatment according to the summary of product characteristics (see Table 3). Additional ADRs $(n=2)$ are included among other reasons (secondary endpoint; see Table 4).

PD: progression of disease; ADRs: adverse events related to bevacizumab.

Table 3. Frequency and type of bevacizumab-related adverse events that led to treatment discontinuation (primary endpoint).

\begin{tabular}{|c|c|}
\hline Adverse events related to bevacizumab & $\begin{array}{l}\text { Patients who discontinued } \\
\text { due to ADRs }(n=22), n(\%)\end{array}$ \\
\hline Pulmonary embolism (grade 4) & $3(12.5)$ \\
\hline Arterial thromboembolism & $2(8.33)$ \\
\hline Pulmonary embolism and lung failure & $2(8.33$ \\
\hline Hypertensive crisis/hypertensive encephalopathy & I (4.16) \\
\hline Hypertension & I (4.16) \\
\hline Healing complications & I (4.16) \\
\hline Grade $3-4$ bleeding & I (4.16) \\
\hline Tracheo-esophageal fistula or any grade 4 fistula & I (4.16) \\
\hline Hypertension not adequately controlled with antihypertensive therapy & I (4.16) \\
\hline Gastrointestinal perforation & I (4.16) \\
\hline Rectal bleeding & I (4.16) \\
\hline Deep venous thrombosis (subclavian, internal jugular, axillary, humeral vein) & I (4.16) \\
\hline Severe lower limb venous thrombosis & I (4.16) \\
\hline Left leg thrombosis & I (4.16) \\
\hline Esophageal ulcer & I (4.16) \\
\hline Pulmonary embolism and lung failure (grade 3) & I (4.16) \\
\hline Deep venous thrombosis & I (4.16) \\
\hline Venous embolism & I (4.16) \\
\hline
\end{tabular}

bevacizumab interruption (i.e. first group: for PD or adverse events [AEs] related to the drug requiring discontinuation of treatment according to SPC; second group: for other reasons) (Table 5). Demographic characteristics were similar across the 2 groups, whereas significant differences were observed with regard to $\mathrm{PS}$ ( $\mathrm{PS}=0$ in $68.9 \%$ and $74.2 \%$ of patients interrupting bevacizumab for PD/ AEs and other reasons, respectively, $\mathrm{PS}=1$ in $29.9 \%$ and $20.4 \%$ and $\mathrm{PS}=2$ in $1.1 \%$ and $5.4 \%$, respectively; $p=$ $.036)$, and the number of organs involved by metastases (1 in $47.7 \%$ vs $70.6 \%$ in the first and second group, respectively; $>1$ in $52.3 \%$ vs $29.4 \%$, respectively, $p=.0001$ ).

The median duration of bevacizumab treatment was 6.7 months (range 0.03-15.4) in patients interrupting for PD, 2.5 months (range 0.03-10.1) for ADRs, and 4.6 months (range 0.03-14.7) for other reasons.

A total of $185(95.9 \%)$ and $54(49.5 \%)$ patients progressed, respectively, in the first and second group of subjects, with a median PFS of 6.9 months (95\% CI 6-7.8) and 13.9 months $(95 \%$ CI 10.7-NA, $p<.0001)$ (Figure 1$)$. In particular, patients interrupting for PD had a PFS of 6.7 months (95\% CI 5.7-7.2), whereas those interrupting for ADRs experienced PD in 63.6\% (14/22) of cases and had a median PFS of 9.5 months (95\% CI 7.5-NA).

\section{Second-line treatment}

A total of $57.9 \%(175 / 302)$ of patients received a secondline treatment: chemotherapy and/or targeted therapy in $97.7 \%(171 / 175)$ of cases. The most common regimen administered was FOLFIRI in $18.9 \%(33 / 175)$ of cases, followed by FOLFOX in $16.0 \%(28 / 175)$. As for chemotherapy plus targeted therapy (given in $28.6 \%$ of cases, $50 / 175)$, FOLFIRI plus cetuximab was the most frequently used regimen $(28.0 \%, 14 / 50)$. Targeted therapy alone was administered in $8.6 \%(15 / 175)$ of patients, whereas $5.7 \%$ 
Table 4. Reasons (other than progression of disease and adverse drug reactions) leading to interruption of first-line bevacizumab (secondary endpoint).

\begin{tabular}{|c|c|}
\hline Reasons & $\begin{array}{l}\text { Eligible patients } \\
(n=386), n(\%)\end{array}$ \\
\hline \multicolumn{2}{|l|}{ Main reasons } \\
\hline $\begin{array}{l}\text { Ro/RI surgical resection } \\
\text { (complete) }\end{array}$ & $15(3.9)$ \\
\hline PS & II (2.8) \\
\hline Achievement of CR & $8(2.1)$ \\
\hline $\begin{array}{l}\text { Complications due to } \\
\text { primary tumor site }\end{array}$ & $8(2.1)$ \\
\hline $\begin{array}{l}\text { Achievement of clinical } \\
\text { response }\end{array}$ & $7(1.8)$ \\
\hline chemotherapy interruption & $6(1.6)$ \\
\hline Patient refusal & $4(1.0)$ \\
\hline Patient compliance & $3(0.8)$ \\
\hline chemotherapy toxicity & $3(0.8)$ \\
\hline RT (in case of rectal cancer) & $2(0.5)$ \\
\hline $\begin{array}{l}\text { Bevacizumab-related } \\
\text { toxicities that did not } \\
\text { require interruption by SPC a }\end{array}$ & $2(0.5)$ \\
\hline Other & $40(10.4)$ \\
\hline \multicolumn{2}{|l|}{ Secondary reasons } \\
\hline No other reasons & $83(2 \mid .5)$ \\
\hline Disease progression & 7 (I.8) \\
\hline RO/RI surgical resection & $5(1.3)$ \\
\hline PS & $2(0.5)$ \\
\hline $\begin{array}{l}\text { chemotherapy tolerability- } \\
\text { toxicity }\end{array}$ & $2(0.5)$ \\
\hline Patient compliance & $\mathrm{I}(0.3)$ \\
\hline Other & $7(1.8)$ \\
\hline NA/NR & $5(1.3)$ \\
\hline
\end{tabular}

Reasons were classified as main or secondary depending on the physician's judgment.

aGrade 2 headache $(n=1)$ and grade 2 hemorrhage $(n=1)$.

R0: complete resection with no microscopic residual tumor; RI: complete resection with no grossly visible tumor as defined by the surgeon, but microscopic cancer may be left behind; PS: performance status; CR: complete response; RT: radiotherapy; SPC: summary of product characteristics; NA: not available; NR: not reported.

$(10 / 175)$ received palliative radiotherapy in the secondline setting.

\section{Safety of bevacizumab administration}

Out of 386 evaluable patients, $13.7 \%(n=53)$ experienced at least one adverse reaction related to bevacizumab during the 15-month follow-up, for a total number of 66 ADRs, the majority $(78.8 \%, 52 / 66)$ of which was resolved without consequences before study end. Furthermore, 22.6\% $(12 / 53)$ of these subjects presented at least one severe $\mathrm{ADR}$ to the $\mathrm{mAb}$. As a consequence, 10 patients were hospitalized or required prolonged hospitalization (all resolved), whereas one patient presented a life-threatening drug-related event.

\section{KRAS mutational status}

To assess whether KRAS status affected the investigated endpoints including safety of bevacizumab, patients were subgrouped according to the presence of mutations. Overall, a KRAS exon 2 mutation was found in $50.8 \%$ (196/386) of cases: of these, $74.0 \%(145 / 196)$ harbored a mutation in codon 12 and $15.3 \%(30 / 196)$ in codon 13. No difference was observed between the 2 groups in terms of demographic and clinical characteristics and chemotherapy regimen administered (data not shown). Also, the median duration of treatment was similar between the 2 groups, being 7.3 months (range $0.03-$ 16.4) and 7.0 months (range 0.03-16.5) in wt vs mut, respectively. Finally, both PD frequency and PFS were comparable between $K R A S$ wt and mut patients undergoing bevacizumab in combination with chemotherapy (PD frequency: $62.6 \%$ vs $65.8 \%$, respectively; PFS: 10.5 months [ $95 \%$ CI 8.7-12.1] vs 10.1 months [95\% CI 9.011.5], respectively).

Patients classified according to KRAS status were further subgrouped based on the reasons for bevacizumab interruption and results reflected those obtained in the whole population. In fact, patients interrupting for PD or ADRs (46.6\% with KRAS wt) vs other reasons $(52.3 \%$ with $K R A S$ wt) more frequently had $>1$ organ affected ( $p=.0123$ in KRAS wt and $p=.0038$ in KRAS mut) and a shorter PFS (data not shown). However, only in the subgroup of patients with KRAS mut, subjects interrupting treatment for PD or ADRs had significantly more synchronous and less metachronous metastases than patients interrupting for other reasons (data not shown).

\section{Discussion}

BEAWARE is the first Italian observational study designed to assess, in the real-world setting, the proportion of patients with mCRC who interrupted first-line bevacizumab over a 15-month period, and to explore in detail the reasons (besides PD and ADRs) and consequences of early discontinuation. Indeed, despite the fact that NCCN guidelines suggest continuing treatment until PD or toxicity, in daily practice bevacizumab is often prematurely interrupted. Our findings show that $78.2 \%$ of eligible patients interrupted $\mathrm{mAb}$ administration in the 15 months of observation, due to $\mathrm{PD}$ in $56.6 \%$ of cases $(44.3 \%)$, bevacizumab-related adverse reaction in $7.3 \%$ (requiring discontinuation of treatment according to SPC: $5.7 \%$ ), or other causes in the remaining $36.1 \%$ (28.2\%). Previous studies have reported an overall frequency of treatment discontinuation of $\sim 60 \%{ }^{17}$ and $71 \%{ }^{4}$ As for causes of interruption, the ARIES observational study indicated PD in $8.3 \%$ of cases, toxicity (AEs or serious AE [SAEs]) in $\sim 30 \%$, and other reasons, including chemotherapy holidays, maximum benefit achieved, planned surgery, and physician's decision, in the remaining. ${ }^{17}$ Instead, in the 
Table 5. Characteristics of patients subgrouped according to the main reason for bevacizumab discontinuation.

\begin{tabular}{|c|c|c|c|}
\hline Variables & $\begin{array}{l}\text { Patients discontinuing for } \\
\text { PD or ADRs }(n=193)\end{array}$ & $\begin{array}{l}\text { Patients interrupting for } \\
\text { other reasons }(n=109)\end{array}$ & $p$ Value \\
\hline Age at diagnosis, $y$ & $62.8 \pm 11.3$ & $63.7 \pm 10.2$ & .50 \\
\hline Age at the start of bevacizumab, $y$ & $63.0 \pm 11.3$ & $63.9 \pm 10.3$ & .5 \\
\hline $\begin{array}{l}\text { Time from diagnosis to start of } \\
\text { bevacizumab, mo }\end{array}$ & $1.9 \pm 2.0$ & $2.0 \pm 2.5$ & .85 \\
\hline $\begin{array}{l}\text { Duration of first-line } \\
\text { bevacizumab, mo }\end{array}$ & $6.3(0.03-15.4)$ & $4.6(0.03-14.7)$ & \\
\hline Male & $116(60.1)$ & $69(63.3)$ & .58 \\
\hline \multicolumn{4}{|l|}{ Concomitant diseases } \\
\hline None & 75 (38.9) & $48(44.0)$ & \\
\hline Hypertension & $69(35.8)$ & $37(33.9)$ & \\
\hline Diabetes & $19(9.8)$ & $7(6.4)$ & \\
\hline Heart disease & $12(6.2)$ & $8(7.3)$ & \\
\hline Dyslipidemia & $10(5.2)$ & $7(6.4)$ & \\
\hline COPD & $5(2.6)$ & $6(5.5)$ & \\
\hline Asthma & $2(1.0)$ & $\mathrm{I}(0.9)$ & \\
\hline Hepatitis B & $0(0.0)$ & $2(1.8)$ & \\
\hline Hepatitis C & $2(1.0)$ & $0(0.0)$ & \\
\hline Renal failure & $2(1.0)$ & $0(0.0)$ & \\
\hline Cirrhosis of liver & I (0.5) & $0(0.0)$ & \\
\hline Other & $6 I(31.6)$ & $31(28.4)$ & \\
\hline ECOG-PS & & & .036 \\
\hline 0 & $122(68.9)$ & $69(74.2)$ & \\
\hline I & $53(29.9)$ & $19(20.4)$ & \\
\hline 2 & $2(I . I)$ & $5(5.4)$ & \\
\hline Organs with metastases, $n$ & & & .0001 \\
\hline I & $92(47.7)$ & $77(70.6)$ & \\
\hline$>1$ & I0I (52.3) & $32(29.4)$ & \\
\hline Type of metastases & & & .23 \\
\hline Synchronous & I I $8(64.1)$ & $58(56.9)$ & \\
\hline Metasynchronous & $66(35.9)$ & $44(43.1)$ & \\
\hline PFS, months (median $[95 \% \mathrm{Cl}]$ ) & $6.9(6.0-7.8)$ & 13.9 (I0.7-NA) & $<.0001$ \\
\hline KRAS mut & $103(53.4)$ & $52(47.7)$ & \\
\hline
\end{tabular}

Statistically significant $(<.05) p$ values are reported in bold. For ECOG-PS and type of metastases, percentages are computed on evaluable patients with available data. For continuous variables, the $t$-test was performed, while for categorical ones, the chi-squared test is provided (Fisher exact test for ECOG-PS). Log-rank test is shown for PFS.

PD: progression of disease; ADR: adverse drug reaction; COPD: chronic obstructive pulmonary disease; ECOG-PS: Eastern Cooperative Oncology Group Performance Status; PFS: progression-free survival; Cl: confidence interval; mut: mutated; NA: not available.

phase III randomized trial conducted by Saltz and coworkers, therapy was discontinued for PD in $29 \%$ of cases and for AEs in approximately $5 \%$ of subjects. ${ }^{4}$ These differences may be partly explained by the study duration, which was 15 months in the present report, 15.6 months in the randomized trial, ${ }^{4}$ and 21 months in the ARIES study. ${ }^{17}$ It is unlikely that the chemotherapy regimens associated with bevacizumab account for the differences observed, as data collected specifically regarded bevacizumab-related AEs. In addition, the sample size of the ARIES study $(n=$ $1550)$ is larger than ours but similar to that of the trial by Saltz et al. $(n=1401) .{ }^{4}$

The median duration of bevacizumab treatment in our study was 7.2 months, whereas previous observational studies reported a duration of $4.7,{ }^{17} 5.4,,^{16} 5.5,{ }^{19}$ and 7 months. ${ }^{20}$ This aspect is of particular interest as some studies suggested an association between the duration of bevacizumab administration and its efficacy, reporting an OS benefit for those patients who continued therapy beyond first PD. ${ }^{14,15,21}$ However, the optimal strategy after first-line chemotherapy plus bevacizumab remains controversial, both in the maintenance ${ }^{11-13}$ and the "beyond progression" setting. ${ }^{14,15}$ While this topic was beyond the focus of the BEAWARE study, in the future it would be important to investigate what happens in Italian daily practice to patients who do not interrupt bevacizumab at PD, both in terms of survival and safety. Finally, median PFS was 10.3 months, in line with previous observational studies reporting a median time to progression between 9.9 and 11.5 months, ${ }^{16,17,19,20,22}$ as well as with randomized 


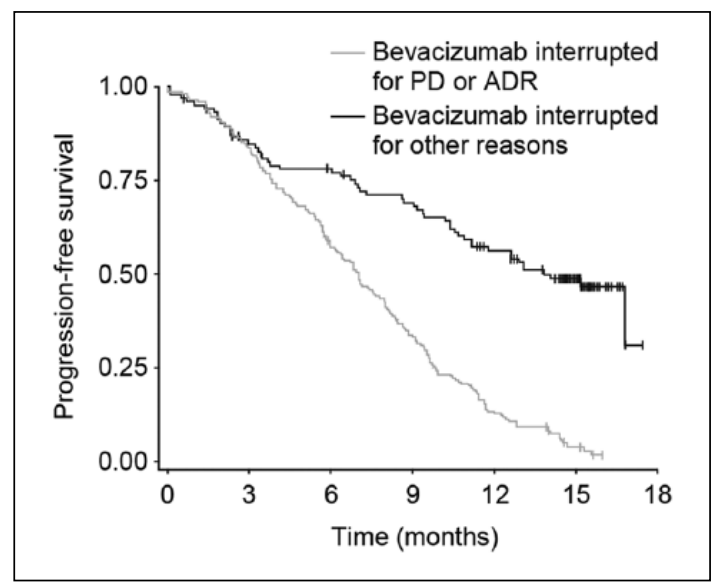

Figure I. Progression-free survival of metastatic colorectal cancer patients subgrouped according to the reasons for bevacizumab discontinuation (progression of disease [PD], adverse drug reactions [ADR], or for other reasons).

trials showing a median PFS ranging from 8.5 to 12.1 months. ${ }^{3,4,23-26}$ In addition, the short period of treatment of patients interrupting for ADR in our study confirms that bevacizumab toxicity, if present, occurs early during treatment.

When the characteristics of patients subgrouped according to reasons for bevacizumab discontinuation were compared, a single metastasis was found to be less common among subjects interrupting for PD or ADRs, compared to those interrupting for other reasons $(47.7 \%$ and $70.6 \%$, $p=.0001)$. Accordingly, the median PFS was 6.9 months in the first group (6.7 months in case of PD and 9.5 in case of ADRs), vs 13.9 in the second one. Yet this is not surprising, as the first group included patients with progressive disease, while the second one comprised subjects who achieved the maximum clinical response or surgical resection.

Approximately $50 \%$ of patients enrolled in the BEAWARE study carried a KRAS exon 2 mutation. No difference was observed between wt and mut subjects in terms of patient characteristics, chemotherapy regimen, treatment duration, PD frequency, or PFS. It is worth noting that we found a median PFS of 10.5 and 10.1 months in wt and mut, respectively, which is in accordance with the median interval of 10 months reported in both KRAS groups in a recent retrospective study assessing the prognostic impact of these mutations in patients with mCRC $(64 \% \mathrm{wt}$ and $36 \%$ mut) receiving first-line bevacizumab. ${ }^{27}$

When patients classified by KRAS status were further subgrouped based on the reason for treatment discontinuation, few differences were found, which mostly reflected those of the whole cohort.

The BEAWARE study has several limitations, some intrinsic to observational studies, such as the lack of randomization. However, participating sites $(n=74)$ were well-distributed among geographic areas and types of institution. Also, patients were consecutively enrolled at each center, taking into account their mutational status, in a proportion of $1: 1$.

The period of observation of the present trial was 20122013; at that time, results of maintenance trials ${ }^{11-13}$ were still not available, and decision on how long to prosecute treatment with bevacizumab in daily practice was made case by case by the physician. As a general message, maintenance trials showed a PFS benefit in continuing bevacizumab, preferably with a fluoropyrimidine, until progression, but a limited impact on survival. Up to now, the impact of those findings in Italian clinical practice is not clear, or if, according to that, the results of the BEAWARE Study are still reproducible after 5 years.

Any consideration on PFS should be taken with caution, due to the short period of observation. In fact, $21.8 \%$ of patients were still receiving bevacizumab and $35.8 \%$ were not progressed at 15 months. All those patients were thus censored for PFS at the last observation, resulting in a potentially immature PFS estimate.

In the BEAWARE study, the most frequent reason for treatment discontinuation was PD, rather than ADRs or other causes. Indeed, bevacizumab-related AEs and SAEs were reported in $13.7 \%$ and $3.1 \%$ of all eligible subjects, respectively, but only about half of them interrupted therapy because of these $(n=24)$, confirming an acceptable safety profile of this agent. In addition, a total of $23(7.6 \%)$ of the patients interrupted bevacizumab due to tumor response (radical surgery or complete response). To avoid an improper interruption in the remaining $26 \%$ (not PD or ADRs, not complete response), a better knowledge on relative contraindications of antiangiogenic therapy that require only temporary withdrawal of the treatment and on the management of concomitant illness should be implemented.

Given the role of continuous VEGF inhibition in firstline treatment, ${ }^{2}$ the attitude to continue bevacizumab until progression in patients with a good balance between benefit and risk might be implemented in Italian clinical practice.

\section{Acknowledgements}

Emanuela Proietti is an employee at Roche SpA Italy. The authors thank the patients who participated in this study and the following investigators (The BEAWARE study group) for their contribution: V. Adamo (Ospedale Papardo-Piemonte, Messina); M. Aieta (Ospedale Oncologico Regionale, Rionero in Vulture, Potenza); O. Alabiso (A.O.U. Maggiore della Carità, Novara); D. Amoroso (Ospedale nuovo della Versilia, Viareggio); F. Angelini (Ospedale Regina Apostolorum-S.C., Albano Laziale, Rome); A. Ardizzoia (Ospedale Di Circolo A. Manzoni, Lecco); C. Aschele (Ospedale Felettino, La Spezia); M. Astorino (Ospedale della Misericordia, Grosseto); M. Benasso (Ospedale Civile, Savona); G. Beretta (Humanitas Gavazzeni, Bergamo); C. Bonciarelli (ULSS 17 Schiavonia, Padua); A. Bonetti (Ospedale Mater Salutis, Legnago, Verona); S. Brugnatelli (Fondazione IRCCS Policlinico San Matteo, Pavia); E. Cammiluzzi (Ospedale Sandro Pertini, Rome); 
F. Cappuzzo (Ospedale Civile, Livorno); M.A. Carapezza (Ospedale degli Infermi, Biella); C. Carlomagno (Univerità degli Studi di Napoli, Naples); M. Cazzaniga (Azienda Ospedaliera S. Gerardo di Monza); F. Ciardiello (Policlino Universitario, Napoli); S. Cinieri (Ospedale Antonio Perrino, Brindisi); A. Comandone (Presidio Sanitario Gradenigo, Torino); L. Crino (Azienda Ospedaliera Di Perugia Ospedale s. Maria Della Misericordia, Perugia); B. Daniele (Ospedale G. Rummo, Benevento); A. D'Arco (Presidio Ospedaliero Umberto I, Nocera Inferiore, Salerno); D. Ferrari (Ospedale San Paolo, Milano); F. Ferrau (Ospedale San Vincenzo, Taormina); C. Ficorella (ASL 4-Osp. S. Salvatore, L'Aquila); I. Fioroni (Policlinico Universitario Campus Biomedico, Rome); G. Francini (Azienda Ospedaliera Universitaria Senese-Policlinico Le Scotte, Siena); A. Frassoldati (Arcispedale S. Anna, Ferrara); M. Giordano (Ospedale S. Anna, Como); L. Giustini (Ospedale Civile Murri, Fermo); O. Gottardi (Clinica Multimedica Holding SpA, Milan); E. Greco (Ospedale di Lamezia Terme, Catanzaro); V.R. Iaffaioli (Gastrointestinal Medical Oncology Unit, Istituto Nazionale per lo Studio e la Cura dei Tumori "Fondazione Giovanni Pascale"-IRCCS, Naples); M.G. Lanzillo (Ospedale Businco, Cagliari); L. Latini (Ospedale, Macerata); V. Leonardi (Ospedale Oncologico Maurizio Ascoli, Palermo); I. Lolli (Azienda Ospedaliera Saverio De Bellis IRCCS, Castellana Grotte, Bari); G. Luppi (A.O. Universitaria Policlinico, Modena); A. Maestri (Ospedale Nuovo, Imola, Bologna); E. Maiello (IRCCS Ospedale Casa Sollievo Della Sofferenza, San Giovanni Rotondo, Foggia); R. Maisano (Azienda Ospedaliera Bianchi Melacrino Morelli, Reggio Calabria); R. Mattioli (Ospedale S. Croce, Fano); M. Mauri (Ospedale San Giovanni Addolorata, Rome); F. Negri (Azienda Ospedaliera Universitaria, Parma); C. Oliani (Ospedale di Montecchio Maggiore, Montecchio Maggiore, Vicenza); S. Palazzo (Ospedale Mariano Santo, Cosenza); A. Pancotti (Ospedale Mazzini, Teramo); F. Pasini (Ospedale S. Maria della Misericordia, Rovigo); I. Pavese (Ospedale San Pietro Fatebenefratelli, Rome); L. Pavesi (Fondazione Salvatore Maugeri, Genoa); G. Pinotti (ASST Sette Laghi, Varese); G. Romano (Ospedale Vito Fazzi, Lecce); S. Romito (Az. Osp. Univ. Ospedali Riuniti, Foggia); F. Rosetti (Ospedale Civile, Mirano); G. Rosti (Ospedale Cà Foncello, Treviso); E. M. Ruggeri (Ospedale Belcolle, Viterbo); H. Soto Parra (Azienda Ospedaliero-Universitaria "Policlinico Vittorio Emanuele,” P.O. G. Rodolico, Catania); M. Spada (Fond. Ist. S. Raffaele G. Giglio, Cefalù); M. Tampellini (A.O.U. San Luigi Gonzaga, Orbassano,Torino); G. Tomasello (Istituti Ospitalieri, Cremona); S. Tumulo (Azienda Ospedaliera S. Maria degli Angeli, Pordenone); C. Verusio (Ospedale Generale Provinciale, Saronno, Varese); V. Zagonel (Istituto Oncologico Veneto-IRCCS, Padua); A. Zaniboni (Fondazione Poliambulanza, Istituto Ospedaliero, Brescia).

\section{Declaration of conflicting interests}

The authors declare that there is no conflict of interest.

\section{Funding}

The author(s) disclosed receipt of the following financial support for the research, authorship, and/or publication of this article: This study was sponsored by Roche SpA, Italy. Scientific and technical coordination, data management, and statistical support were provided by Medidata Observational Research, Italy.
Medical writing support and editorial assistance was provided by Clara Ricci, PhD (Primula Multimedia SrL, Pisa) and funded by Roche (no grant number).

\section{References}

1. Ferlay J, Steliarova-Foucher E, Lortet-Tieulent J, et al. Cancer incidence and mortality patterns in Europe: estimates for 40 countries in 2012. Eur J Cancer 2013; 49: 1374-1403.

2. Van Cutsem E, Cervantes A, Adam R, et al. ESMO consensus guidelines for the management of patients with metastatic colorectal cancer. Ann Oncol 2016; 27: 1386-422.

3. Hurwitz H, Fehrenbacher L, Novotny W, et al. Bevacizumab plus irinotecan, fluorouracil, and leucovorin for metastatic colorectal cancer. N Engl J Med 2004; 350: 2335-2342.

4. Saltz LB, Clarke S, Díaz-Rubio E, et al. Bevacizumab in combination with oxaliplatin-based chemotherapy as firstline therapy in metastatic colorectal cancer: a randomized phase III study. J Clin Oncol Off J Am Soc Clin Oncol 2008; 26: 2013-2019.

5. Hochster HS, Hart LL, Ramanathan RK, et al. Safety and efficacy of oxaliplatin and fluoropyrimidine regimens with or without bevacizumab as first-line treatment of metastatic colorectal cancer: results of the TREE Study. J Clin Oncol 2008; 26: 3523-3529.

6. Van Cutsem E, Lenz HJ, Köhne $\mathrm{CH}$, et al. Fluorouracil, leucovorin, and irinotecan plus cetuximab treatment and RAS mutations in colorectal cancer. J Clin Oncol 2015; 33: 692-700.

7. Maughan TS, Adams RA, Smith CG, et al. Addition of cetuximab to oxaliplatin-based first-line combination chemotherapy for treatment of advanced colorectal cancer: results of the randomised phase $3 \mathrm{MRC}$ COIN trial. Lancet Lond Engl 2011; 377: 2103-2114.

8. Douillard JY, Siena S, Cassidy J, et al. Final results from PRIME: randomized phase III study of panitumumab with FOLFOX4 for first-line treatment of metastatic colorectal cancer. Ann Oncol 2014; 25: 1346-1355.

9. Cutsem EV, Tabernero J, Lakomy R, et al. Addition of aflibercept to fluorouracil, leucovorin, and irinotecan improves survival in a phase III randomized trial in patients with metastatic colorectal cancer previously treated with an oxaliplatinbased regimen. J Clin Oncol 2012; 30: 3499-3506.

10. Tabernero J, Yoshino T, Cohn AL, et al. Ramucirumab versus placebo in combination with second-line FOLFIRI in patients with metastatic colorectal carcinoma that progressed during or after first-line therapy with bevacizumab, oxaliplatin, and a fluoropyrimidine (RAISE): a randomised, double-blind, multicentre, phase 3 study. Lancet Oncol 2015; 16: 499-508.

11. Simkens LHJ, van Tinteren H, May A, et al. Maintenance treatment with capecitabine and bevacizumab in metastatic colorectal cancer (CAIRO3): a phase 3 randomised controlled trial of the Dutch Colorectal Cancer Group. Lancet 2015; 385: 1843-1852.

12. Hegewisch-Becker S, Graeven U, Lerchenmüller CA, et al. Maintenance strategies after first-line oxaliplatin plus fluoropyrimidine plus bevacizumab for patients with metastatic colorectal cancer (AIO 0207): a randomised, non-inferiority, open-label, phase 3 trial. Lancet Oncol 2015; 16: 1355-1369. 
13. Koeberle D, Betticher DC, von Moos R, et al. Bevacizumab continuation versus no continuation after first-line chemotherapy plus bevacizumab in patients with metastatic colorectal cancer: a randomized phase III non-inferiority trial (SAKK 41/06). Ann Oncol 2015; 26: 709-714.

14. Bennouna J, Sastre J, Arnold D, et al. Continuation of bevacizumab after first progression in metastatic colorectal cancer (ML18147): a randomised phase 3 trial. Lancet Oncol 2013; 14: 29-37.

15. Masi G, Salvatore L, Boni L, et al. Continuation or reintroduction of bevacizumab beyond progression to firstline therapy in metastatic colorectal cancer: final results of the randomized BEBYP trial. Ann Oncol 2015; 26: 724-730.

16. Kozloff M, Yood MU, Berlin J, et al. Clinical outcomes associated with bevacizumab-containing treatment of metastatic colorectal cancer: the BRiTE observational cohort study. Oncologist 2009; 14: 862-870.

17. Bendell JC, Bekaii-Saab TS, Cohn AL, et al. Treatment patterns and clinical outcomes in patients with metastatic colorectal cancer initially treated with FOLFOX-bevacizumab or FOLFIRI-bevacizumab: results from ARIES, a bevacizumab observational cohort study. Oncologist 2012; 17: 1486-1495.

18. Russo AL, Borger DR, Szymonifka J, et al. Mutational analysis and clinical correlation of metastatic colorectal cancer. Cancer 2014; 120: 1482-1490.

19. Fourrier-Réglat A, Smith D, Rouyer M, et al. Survival outcomes of bevacizumab in first-line metastatic colorectal cancer in a real-life setting: results of the ETNA cohort. Target Oncol 2014; 9: 311-319.

20. Stein A, Petersen V, Schulze M, et al. Bevacizumab plus chemotherapy as first-line treatment for patients with metastatic colorectal cancer: results from a large German community-based observational cohort study. Acta Oncol Stockh Swed 2015; 54: 171-178.

21. Grothey A, Sugrue MM, Purdie DM, et al. Bevacizumab beyond first progression is associated with prolonged overall survival in metastatic colorectal cancer: results from a large observational cohort study (BRiTE). J Clin Oncol Off $J$ Am Soc Clin Oncol 2008; 26: 5326-5334.

22. Buchler T, Pavlik T, Melichar B, et al. Bevacizumab with 5-fluorouracil, leucovorin, and oxaliplatin versus bevacizumab with capecitabine and oxaliplatin for metastatic colorectal carcinoma: results of a large registry-based cohort analysis. BMC Cancer 2014; 14: 323.

23. Kabbinavar FF, Schulz J, McCleod M, et al. Addition of bevacizumab to bolus fluorouracil and leucovorin in firstline metastatic colorectal cancer: results of a randomized phase II trial. J Clin Oncol 2005; 23: 3697-3705.

24. Tebbutt NC, Wilson K, Gebski VJ, et al. Capecitabine, bevacizumab, and mitomycin in first-line treatment of metastatic colorectal cancer: results of the Australasian Gastrointestinal Trials Group Randomized Phase III MAX Study. J Clin Oncol 2010; 28: 3191-3198.

25. Petrelli F, Borgonovo K, Cabiddu M, et al. FOLFIRIbevacizumab as first-line chemotherapy in 3500 patients with advanced colorectal cancer: a pooled analysis of 29 published trials. Clin Colorectal Cancer 2013; 12: 145151.

26. Loupakis F, Cremolini C, Masi G, et al. Initial therapy with FOLFOXIRI and bevacizumab for metastatic colorectal cancer. N Engl J Med. 2014; 371: 1609-1618.

27. Rossi L, Veltri E, Zullo A, et al. Metastatic colorectal cancer first-line treatment with bevacizumab: the impact of K-ras mutation. OncoTargets Ther 2013; 6: 1761-1769. 